\title{
Neuere mathematische Formulierungen der biologischen Temperaturfunktion und des Wachstums
}

\author{
FrIEDRICH KRÜGER \\ Biologische Anstalt Helgoland, Zentrale, Hamburg-Altona
}

\begin{abstract}
Newer mathematic expressions of the biological temperature function and of growth. The effects of temperature on metabolic rates cannot be assessed satisfactorly on the basis of $\mathrm{Q}_{10}$-values or by the ARRHENIUs formula. In many cases a surprisingly exact expression is obtained if a lower temperature value is used in the power of the denominator of the ARRHENIUS-formula instead of the reciprocal value of the absolute temperature. An analysis of Krogh's data shows that the examples upon which he based his "standard-curve" may be expressed very appropriately by the new expression presented in this paper. By using principally the same formula it becomes possible to express growth rates (in units of weight or length) of different kinds of animals. Its parameters allow to deduce the parameters of the allometric formula. The new formula seems to be suitable for expressing values of metabolic rates as a function of age. Considerations concerning the results of Jos (1955) on the trout Salvelinus fontinalis demonstrate that it is principally also possible to express mathematically changes of temperature-rate functions during growth.
\end{abstract}

\section{EINLEITUNG}

In jeder Stunde wächst die Zahl physiologischer Meßwerte, die zahlenmäßige Beziehungen zwischen verschiedenen Meßgrößen liefern. Wenn wir nicht Gefahr laufen wollen, daß in naher Zukunft unsere wissenschaftliche Arbeit sinnlos wird, weil wir ihre Ergebnisse nicht mehr übersehen, können, müssen wir Mittel und Wege finden, sie wenigstens näherungsweise durch adäquate mathematische Methoden darzustellen. So wertvoll eine statistische Verarbeitung im einzelnen ist, so gestattet sie doch nur unsere Meßergebnisse mathematisch einwandfrei anzugeben und ihre Zuverlässigkeit zu prüfen. Die Statistik liefert $z$ war für den Einzelfall Aufschluß über geprüfte Beziehungen, bietet aber nur in beschränktem Umfang eine Möglichkeit, allgemeingültige $\mathrm{Zu}$ sammenhänge aufzudecken.

Stellen wir die im Experiment gefundenen, zahlenmäßigen Beziehungen graphisch dar, so erhalten wir sehr verschieden gestaltete Kurven, deren Verlauf wir nur selten direkt durch mathematische Formulierungen wiedergeben können. Daher liegt vor uns die Aufgabe, nach mathematischen Kennzahlen zu suchen, die geeignet sind, den im Experiment gefundenen Kurvenverlauf zu charakterisieren. Glückt uns dieses, so können wir nicht nur die Darstellung unserer Versuchsergebnisse vereinfachen, sondern gewinnen außerdem exakte Unterlagen für vergleichende Betrachtungen. Die moder- 
nen Rechenmittel - mechanische und elektronische - machen es heute für den Biologen nicht mehr zu einer kaum zu lösenden Aufgabe, kompliziertere Formeln selbst zu berechnen oder nötigenfalls berechnen zu lassen. Hiermit ist ein wichtiges Hemmnis entfallen, das bislang einer mathematischen Verarbeitung biologischer Ergebnisse im Wege stand.

Das Problem, vor dem der Biologe noch steht, ist die mathematische Analyse komplizierter Kurvenverläufe, die der Mathematiker nur ungerne und dann auch nur mit rein mathematischen Methoden durchführt. Hierbei kommt der Mathematiker oft zu Lösungen, die dem Biologen keinen Nutzen bringen. Nur in Ausnahmefällen haben wir Biologen die Möglichkeit, von mathematisch erfaßten Elementarvorgängen auszugehen und aus diesen die Funktion für die gefundene Kurve zu entwickeln. In der Mehrzahl der Fälle müssen wir versuchen, auf anderen Wegen die zur Darstellung unserer Ergebnisse geeigneten mathematischen Formulierungen zu finden. Einen Weg, auf dem dieses Problem in gee"gneten Fällen gelöst werden kann, möchte ich heute aufzeigen und die Ergebnisse a cinigen Beispielen besprechen.

Häufig ist man in der Biologie bei der mathematischen Darstellung in der Weise vorgegangen, daß man versuchte, entsprechende Formulierungen aus dem Bereich der anorganischen Wissenschaften auf die eigenen Probleme anzuwenden. Ein typisches Beispiel stellt die Darstellung der Temperaturfunktion biologischer Vorgänge durch die Formel von BERTHELOT (1862)

$$
y=a \cdot b^{t}
$$

in Form des von VAN'T HoFf vorgeschlagenen $Q_{10}$-Wertes dar. Ungeachtet der Tatsache, daß dieser Wert keine mathematisch befriedigende Darstellung biologischer Temperaturfunktionen gestattet, ist er bis zum heutigen Tage noch in Gebrauch. Auch die im Prinzip auf VAN'T Hoff (1884) zurückgehende Temperaturfunktion von ARRHENIUS (1889)

$$
y=y_{\max } \cdot e^{-} R \cdot \frac{\mu}{(t+273)}
$$

unterscheidet sich in ihrem Kurvenverlauf - aus mathematisch leicht ersichtlichen Gründen - im Bereich biologischer Temperaturen nicht erheblich von der BERTHELOTFunktion. Sie gestatter daher in der Mehrzahl der Fälle keine bessere Wiedergabe biologischer Meßwerte. Schon vor nahezu 50 Jahren erkannte KRogh (1914) diese Tatsache und versuchte auf Grund eigener sehr sorgfältiger Messungen eine Standardkurve aufzustellen, die seine Ergebnisse näherungsweise wiedergab. Die damals von ihm entworfene Kurve dient auch heute noch als Bezug für viele Untersuchungen. KROGH selbst hat keinen Versuch unternommen, für seine Kurve eine Formel aufzustellen. Ein mathematisch nicht ungeeigneter Versuch von JøRGENSEN (1916) ist in der Biologie unbeachtet geblieben. Ein einfacher Kurvenvergleich schließt aber soviel subjektive Momente ein, daß er für eine Auswertung von Versuchsergebnissen ungeeignet ist. Aus diesem Grunde bemühte ich mich, für eigene Meßreihen, die im Prinzip die KroGHsche Standardkurve lieferten, eine exaktere mathematische Formulierung zu finden. 


\section{ABLEITUNG UND AUSWERTUNG DER FUNKTIONEN}

Stellt man in einem semilogarithmischen Koordinatensystem die Logarithmen der Stoffwechselgröße den linearen Temperaturwerten gegenüber, so müßten im Falle des Zutreffens der $Q_{10}$-Regel die Meßwerte in der Nähe einer Geraden angeordnet sein. Bekanntlich liegen aber im allgemeinen die Meßpunkte bei dieser Art der Darstellung auf einer Kurve, die mit zunehmender Temperatur sich immer weiter von der Geraden entfernt. Die Kurve verändert nur wenig ihren Verlauf, wenn man entsprechend der ARrHENIUS-Formulierung als Abscissenteilung den reziproken Wert der absoluten Temperatur wählt. Das im Vergleich zu dem in Frage kommenden Temperaturbereich hohe additive Glied im Nenner des Exponenten hat nur geringen Einfluß auf die Temperaturabstände in der Abscissen-Teilung, die nahezu gleich bleiben.

Benutzt man als Abscissenteilung nicht den reziproken Wert der absoluten Temperatur, sondern den reziproken Wert der einfachen Celsiusgrade - also anstatt $1 /(t+273)$ den Wert $1 /(t+0)-$, so erhält man eine Kurve, die den entgegengesetzten

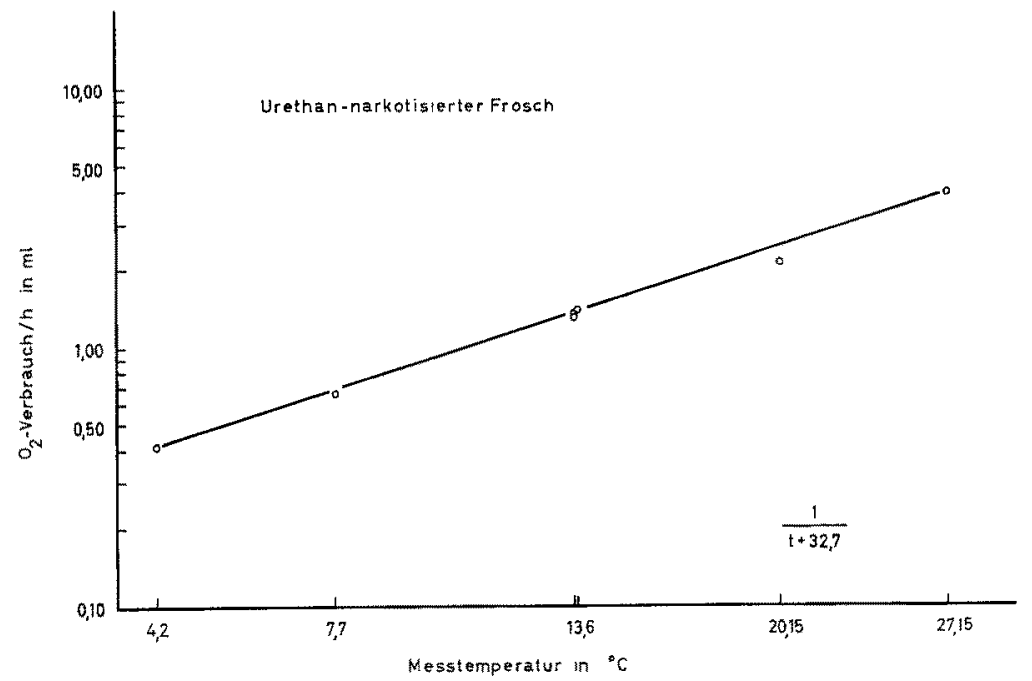

Abb. 1: Graphische Darstellung der Meßwerte von KroGH für den Urethan-narkotisierten Frosch als Funktion von $1 / t+32,7$

Krümmungssinn hat wie die ArRHENrus-Kurve. Die beiden Kurven öffnen sich gegeneinander. Hieraus schloß ich, daß mit einem Temperaturwert, der zwischen 0 und 273 liegt, sich die Meßpunkte wenigstens näherungsweise durch eine Gerade darstellen lassen müssen.

Das Ergebnis bestätigte die Erwartung. Ich mödhte es an KRoGHs eigenen Beispielen erläutern (KRÜGER 1963b). Die Abbildungen 1 bis 3 zeigen seine Meßwerte für Frosch, Goldfisch und Hund, dargestellt durch geeignete additive Temperaturwerte. Sogar die abweichend verlaufende Temperaturfunktion der Tenebrio-Puppen (Abb. 4) läßt sich auf diese Weise hervorragend darstellen. Aus der Art der graphischen Darstellung ergibt sich die Formel der biologischen Temperaturfunktion zu: 


$$
\mathrm{y}_{\mathrm{t}}=\frac{\mathrm{m}}{\mathrm{n}^{\mathrm{t}-\mathrm{z}}}
$$

$z$ stellt in der Gleichung die additive (Nullpunkt) Temperatur dar. Da z im allgemeinen negativ ist, muß sein Wert zu der gemessenen Temperatur addiert werden; $m$ und $n$ sind Parameter, die ebenso wie $z$ aus den Meßwerten berechnet werden können. Auf die Wege zu ihrer Bestimmung will ich an dieser Stelle nicht eingehen. Relativ leicht

Tabelle 1

Vergleich der Meß̂werte von Ege \& Krogh (1914) für den Goldfisch Carassius auratus) mit

\begin{tabular}{|c|c|c|c|}
\hline${ }^{\text {Temperatur }}$ & $\begin{array}{l}\text { Meßwerte } \\
\text { O }- \text { Verbrauch } \\
\mathrm{m} / / \mathrm{h} / 9,3 \mathrm{~g}\end{array}$ & $\begin{array}{c}\text { Berechnete Werte } \\
\mathrm{O}_{2} \text {-Verbrauch } \mathrm{m} 1 / \mathrm{h} / 9,3 \mathrm{~g} \\
\mathrm{z}=-34,4 ; \log \mathrm{n}=116,0 \\
\mathrm{~m}=142,49\end{array}$ & $\begin{array}{c}\text { Abweichung } \\
0 / 0\end{array}$ \\
\hline 0 & 0,062 & 0,061 & $-1,61$ \\
\hline 0 & 0,060 & 0,061 & $+\quad 1,67$ \\
\hline 7,9 & 0,263 & 0,260 & $-1,14$ \\
\hline 7,9 & 0,331 & 0,260 & $-21,45$ \\
\hline 14,4 & 0,608 & 0,601 & $-1,15$ \\
\hline 14,9 & 0,614 & 0,636 & $\begin{array}{l}+3,58 \\
+\end{array}$ \\
\hline 15,0 & 0,675 & 0,643 & $-4,74$ \\
\hline 20,1 & 1,010 & 1,065 & $+5,45$ \\
\hline 20,1 & 1,038 & 1,065 & $+2,60$ \\
\hline 24,8 & 1,635 & 1,570 & $-3,98$ \\
\hline 25,5 & 1,646 & 1,655 & $+0,55$ \\
\hline 25,7 & 1,641 & 1,680 & $\begin{array}{r}2,38 \\
+\quad 2,38\end{array}$ \\
\hline 25,7 & 1,735 & 1,680 & $-3,17$ \\
\hline
\end{tabular}
den nach Gleichung (3) berechneten Werten

lassen sie sich näherungsweise durch die graphische Darstellung ermitteln. In Tabelle 1 sind die auf diese Weise berechneten Werte für die Temperaturabhängigkeit der Goldfisch-Atmung den gefundenen Werten gegenübergestellt.

Die hier vorgeschlagene Temperaturfunktion besitzt vor anderen möglichen Formulierungen den großen Vorzug, auch die ARRHENius-Formel einzuschließen und damit auch näherungsweise die RGT-Regel. Wichtiger als die hier aufgezeigte Lösung eines speziellen Problems dürte der an diesem Beispiel aufgezeigte Weg zu seiner Lösung sein. Dieses Verfahren läuft darauf hinaus, bei der graphischen Darstellung durch Variation der Koordinaten-Teilung die ursprïngliche Kurve in eine Gerade überzuführen. Durch die gewählten Koordinatenteilungen ist der mathematische Zusammenhang der geprïften Größen gegeben und gestattet die Aufstellung einer geeigneten Formel.

Naturgemäß liefert das hier vorgeschlagene Verfahren zur mathernatischen Analyse von Kurvenverläufen nur eine rein deskriptive Formel. Das ausschlaggebende Kriterium für ihre Anwendbarkeit bleibt dann aber weniger die exakte Wiedergabe von einzelnen Zahlenreihen, als der Bereich, für den sie gültig ist und in welchem Umfang sich mathematische Beziehungen zu anderen bekannten. Funktionen herstellen lassen. Sind diese Forderungen erfült, iberschreitet die aufgedeckte mathematische Funktion den Rahmen einer reinen Beschreibung. 
Die mathematische Bearbeitung der Temperaturfunktionen führte mich auch auf das Problem der mathematischen Darstellung des tierischen Wachstums als Funktion der Zeit. Ich versuchte es unabhängig von den in der Literatur gemachten Vorschlägen rein deskriptiv in der geschilderten Weise zu lösen.

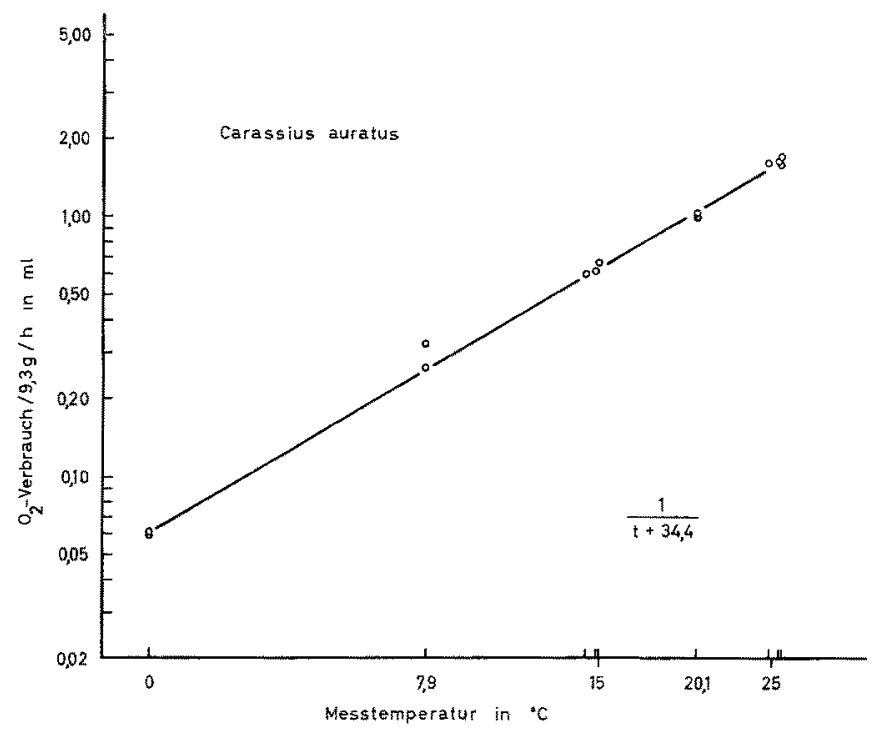

Abb. 2: Darstellung der Meßwerte für den Goldfisch (EGE \& KROGH 1914) als Funktion von $1 / t+34,4$

$\mathrm{Zu}$ meinem größten Erstaunen zeigte sich, daß prinzipiell die gleiche Funktion, die ich zur Darstellung der Temperaturfunktion angewandt hatte, sich auch hervorragend zur Wiedergabe von Wachstumsdaten eignet (KR ÜGER 1962). Im Gegensatz zu den bislang vorgeschlagenen Formeln eignet sich die neue Formel ebensogut zur Darstellung des Gewichts- wie des Längenwachstums, die also auf diesem Wege durch die gleiche Funktion erfaßt werden können. Der neuen Wachstumsfunktion gab ich die Form:

$$
\mathrm{y}_{\chi}=\frac{\mathrm{D}_{\max }}{\mathrm{N}^{\gamma+\zeta}}
$$

In der neuen Formel bedeutet $\chi$ das postnatale Alter, also $y_{\chi}$ die Dimension im Zeitpunkt $\chi_{*}$. Mit $\zeta$ bezeichne ich einen zum Geburtsalter zu addierenden Zahlenwert, der mathematisch ein praenatales Alter darstellt. Das in die Berechnungen eingehende Gesamtalter des Organismus ist also gegeben durch den Wert $\chi+\zeta$, der sich im Exponenten des Nenners findet. $N$ ist ein Ausdruck für die Wachstumsgeschwindigkeit und $D_{\text {max }}$ ist mathematisch die Maximal-Dimension, die der Organismus bei unendlichem unbegrenztem Wachstum erreicht. Wie weit diesen zunächst rein mathematischen Zahlenwerten eine biologische Bedeutung zukommt, läßt sich heute noch nicht übersehen. 
Zunächst möchte ich die Anwendung der neuen Wachstumsformel an einem Beispiel erläutern, und zwar am Thunfisch (Thunnus thynnus), für den über einen Zeitraum von 14 Jahren sehr gut übereinstimmende Zahlen verschiedener Untersucher für

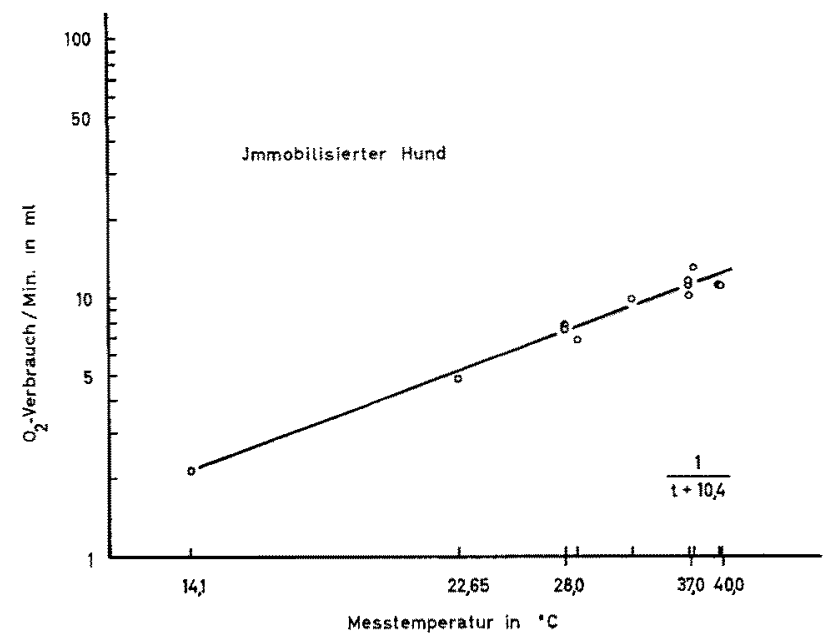

Abb. 3: Graphische Darstellung der Meßwerte von Krogh für den immobilisierten Hund als Funktion von $1 / t+10,4$

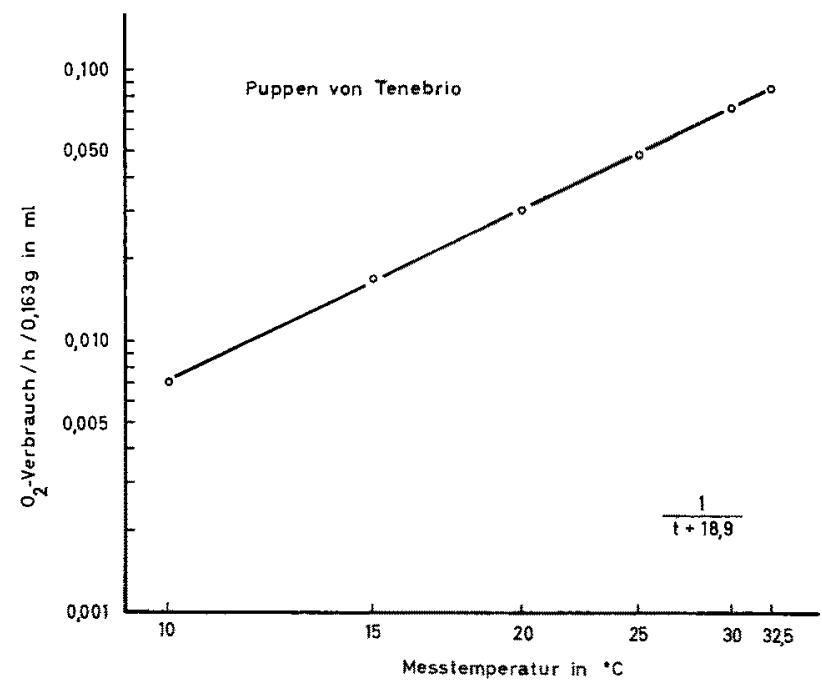

Abb. 4: Graphische Darstellung der Meßwerte von KroGH für die Mehlwurm-Puppen als Funktion von $1 / t+18,9$

das Gewichts- und Längenwachstum vorliegen (Sella 1929). Wir können das Wachstum des Thunfisches in beiden Dimensionen graphisch im gleichen Koordinatensystem als Geraden darstellen, wenn wir die Logarithmen der Dimensionen dem reziproken Wert des um 7 erhöhten Alters gegenüberstellen (Abb. 5). 


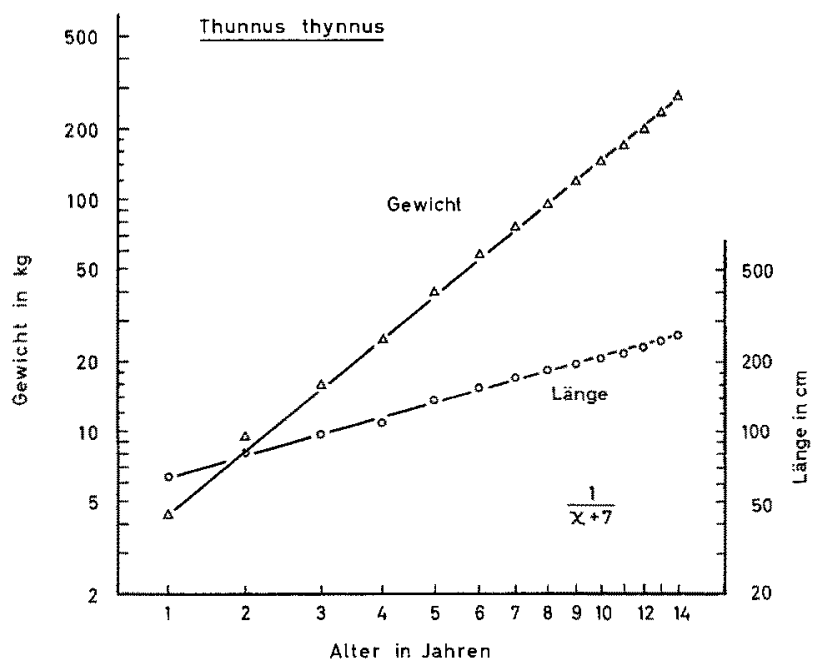

Abb. 5: Graphische Darstellung des Längen- und Gewichtswachsturns des Thunfisches (SELlA 1929) als Funktion des reziproken Wertes des um $7(\xi)$ exhöhten Alters $(\chi)$ in Jahren

Der Wert des additiven Alters ist nicht sehr kritisch. Rechnerisch ergibt sich für das Längenwachstum ein Wert von 6,1, für das Gewichtswachstum ein Wert von 7,3. Bei der Schwankungsbreite und Unsicherheit, mit der alle Wachstumsdaten behaftet sind, ist es heute noch nicht möglich, für das praenatale Alter mathematisch ganz ein-

Tabelle 2

Vergleich der Längen- und Gewichtsangaben von SeLLA (1929) für die Altersklassen 1-14 des Thunfisches (Thunnus thynnus) mit den nach Gleichung (4) berechneten Werten

\begin{tabular}{|ccccccc|}
\hline $\begin{array}{c}\text { Alters- } \\
\text { klasse }\end{array}$ & gemessen & $\begin{array}{c}\text { Länge in cm } \\
\text { berechnet }\end{array}$ & $\begin{array}{c}\text { Abweichung } \\
0 \%\end{array}$ & gemessen & Gewicht in kg \\
berechnet & $\begin{array}{c}\text { Abweichung } \\
\text { \% }\end{array}$ \\
\hline 1 & 64 & 63,79 & $-0,33$ & 4,4 & 4,37 & $-0,58$ \\
2 & 81,5 & 81,70 & $+0,24$ & 9,5 & 9,14 & $-3,80$ \\
3 & 97,5 & 99,58 & $+2,13$ & 16 & 16,48 & $+2,97$ \\
4 & 118 & 117,08 & $-0,78$ & 25 & 26,68 & $+6,72$ \\
5 & 136 & 134,01 & $-1,46$ & 40 & 39,89 & $-0,29$ \\
6 & 153 & 150,21 & $-1,82$ & 58 & 56,04 & $-3,39$ \\
7 & 169 & 165,65 & $-1,98$ & 76 & 74,98 & $-1,35$ \\
8 & 182 & 180,30 & $-0,93$ & 95 & 96,51 & $+1,59$ \\
9 & 195 & 194,21 & $-0,41$ & 120 & 120,40 & $+0,33$ \\
10 & 206 & 207,37 & $+0,67$ & 145 & 146,35 & $+0,93$ \\
11 & 216 & 219,76 & $+1,74$ & 170 & 173,98 & $+2,34$ \\
12 & 227 & 231,54 & $+2,00$ & 200 & 203,23 & $+1,62$ \\
13 & 239 & 242,64 & $+1,52$ & 235 & 233,65 & $-0,57$ \\
14 & 254 & 253,16 & $-0,33$ & 280 & 265,09 & $-5,33$ \\
\multicolumn{7}{c}{ Parameter; } \\
\end{tabular}


deutige Werte einzusetzen. Praktisch ist es aber schon heute möglich, mit Näherungswerten von $\zeta$ einen sehr guten Anschluß an vorliegende Wachstumsdaten zu erreichen.

In Tabelle 2 sind die Werte berechnet, die sich bei dem gleichen Wert für $\zeta$ von 7 für das Längen- und Gewichtswachstum ergeben. Die Abweichungen erreichen nur beim Gewicht zweimal Werte über $5 \%$, sonst liegen sie wesentlich darunter. Auch an zahlreichen anderen Beispielen, auf deren Wiedergabe an dieser Stelle verzichtet werden soll, konnte eine sehr befriedigende Annäherung an Wachstumsdaten erreicht werden.

Tabelle 3

Vergleich der nach den Formeln von v. BerTALANFFY und der nado Gleichung (4) berechneten Längen des Thunfisches (Thunnus thynnus). (Daten aus Sella 1929)

\begin{tabular}{|c|c|c|c|c|c|}
\hline \multirow[b]{2}{*}{$\begin{array}{l}\text { Alters- } \\
\text { klasse }\end{array}$} & \multirow[b]{2}{*}{$\begin{array}{c}\text { Länge } \\
\mathrm{cm}\end{array}$} & \multicolumn{2}{|c|}{ v. BERTALANFFY } & \multicolumn{2}{|c|}{ KRÜGER } \\
\hline & & $\begin{array}{c}\mathrm{L}_{\max }=460 \\
\mathrm{~K} \cdot \log \mathrm{e}=0,0216 \\
\mathrm{t}_{0}=-2,0158\end{array}$ & $\begin{array}{l}\text { Diffe- } \\
\text { renz } \\
\% \%\end{array}$ & $\begin{array}{c}L_{\max }=531 \\
\log N=6,588 \\
\zeta=6,1055\end{array}$ & $\begin{array}{c}\text { Diffe- } \\
\text { renz } \\
0 / 0\end{array}$ \\
\hline 1 & 64 & 64 & \pm 0 & 62,7 & $-2,03$ \\
\hline 2 & 81,5 & 83,2 & $+2,09$ & 81,7 & $+0,25$ \\
\hline 3 & 97,5 & 101,5 & $+4,10$ & 100,3 & $+2,87$ \\
\hline 4 & $118^{\prime}$ & 118,8 & $+0,68$ & 118,4 & $+0,34$ \\
\hline 5 & 136 & 135,4 & $-0,44$ & 135,0 & $-0,74$ \\
\hline 6 & 153 & 151,1 & $-1,24$ & 151,6 & $-0,91$ \\
\hline 7 & 169 & 166,1 & $-1,72$ & 166,1 & $-1,72$ \\
\hline 8 & 182 & 180,3 & $-0,93$ & 181,2 & $-0,44$ \\
\hline 9 & 195 & 193,9 & $-0,56$ & 194,4 & $-0,31$ \\
\hline 10 & 206 & 206,8 & $+0,39$ & 206,9 & $+0,44$ \\
\hline 11 & 216 & 219,1 & $+1,44$ & 218,6 & $+1,11$ \\
\hline 12 & 227 & 230,7 & $-1,63$ & 229,6 & $+1,15$ \\
\hline 13 & 239 & 241,9 & $+1,21$ & 239,9 & $+0,38$ \\
\hline 14 & 254 & 252,4 & $-0,63$ & 249,6 & $-1,73$ \\
\hline
\end{tabular}

Zum Vergleich habe ich auch das Längenwachstum des Thunfisches nach der Wachstumsformel von $v$. BERTALANFFY berechnet (Tab. 3). Hierbei - wie auch in anderen Fällen - konnte ich feststellen, daß die mit ihr gewonnenen Zahlen für das Längenwachstum eine ausgezeichnete Übereinstimmung mit Wachstumsdaten zeigen. Beachtenswerterweise weichen beide Funktionen in gleichem Sinne von den Daten von SELLA ab. Die gleiche Tatsache zeigte sich auch bei der rechnerischen Auswertung einer Zahlenreihe von QUETELET für das menschliche Wachstum (Tab. 4) nach der Formel von v. BERTALANFFy und nach meinem eigenen Vorschlag. Es zeigt sich hier also eine überraschende Parallele zwischen der Wachstumsberechnung nach den beiden Funktionen, die im übrigen auch in der mathematischen Form sehr ähnlich sind, indem die Größe als Bruchteil einer Maximaldimension dargestellt wird. Schreibt man die Formel von v. BERTALANFFy in der von BEVERTON \& HOLT (1957) vorgeschlagenen Fassung,

$$
\mathrm{l}_{\mathrm{t}}=\mathrm{L}_{\max }\left(1-\mathrm{e}^{-\mathrm{k}\left(\mathrm{t}-\mathrm{t}_{0}\right)}\right)
$$

so wird die Parallele noch auffälliger, da sie dem Alter ebenfalls einen Zeitwert $\left(t_{0}\right)$ addiert, der dem $\zeta$-Wert meiner Funktion entspricht.

Bei der ausgezeichneten Eignung der BERTALANFFX-Formel zur Wiedergabe von Wachstumsdaten erhebt sich die Frage: Hat es einen Sinn, einen neuen Formelvorschlag 
Tabelle 4

Vergleich der Berechnung des Längenwachstums (in $\mathrm{cm}$ ) des Menschen - nach einer Zahlenreihe von QueTELET - unter Benutzung der Wachstumsgleichung von v. BERTALANFFY u. Gleichung (4). v. BERTALANFFY: $L_{\max }=250,0 \mathrm{~cm} ; \mathrm{k} \cdot \ln \mathrm{e}=0,0186 ; \mathrm{t}_{0}=-6,644$ KRÜGER: $L_{\max }=333,4 \mathrm{~cm} ; \log \mathrm{N}=9,877 ; \zeta=13,69$

\begin{tabular}{|c|c|c|c|c|c|}
\hline Alter & QUETELET & KRÜGER & $\underset{\%}{\text { Diff. }}$ & v. BERT ALANFFY & $\underset{\%}{\text { Diff. }}$ \\
\hline 1 & 69,8 & 70,9 & $+1,6$ & 69,8 & 0 \\
\hline 2 & 79,1 & 78,2 & $-1,1$ & 77,4 & $-2,1$ \\
\hline 3 & 86,4 & 85,3 & $-1,3$ & 84,6 & $-2,1$ \\
\hline 4 & 92,7 & 92,2 & $-0,5$ & 91,5 & $-1,3$ \\
\hline 5 & 98,7 & 98,7 & 0 & 98,2 & $-0,5$ \\
\hline 6 & 104,6 & 105,5 & $+0,9$ & 104,5 & $-0,1$ \\
\hline 7 & 110,4 & 111,0 & $+0,5$ & 110,6 & $+0,2$ \\
\hline 8 & 116,2 & 116,8 & $+0,5$ & 116,5 & $+0,3$ \\
\hline 9 & 121,8 & 122,3 & $+0,4$ & 122,1 & $+0,3$ \\
\hline 10 & 127,3 & 127,6 & $+0,2$ & 127,4 & $+0,1$ \\
\hline 11 & 132,5 & 132,7 & $+0,2$ & 132,6 & $+0,1$ \\
\hline 12 & 137,5 & 137,5 & 0 & 137,5 & 0 \\
\hline 13 & 142,3 & 142,2 & $-0,1$ & 142,2 & $-0,1$ \\
\hline 14 & 146,9 & 146,6 & $-0,2$ & 146,7 & $-0,1$ \\
\hline 15 & 151,3 & 150,9 & $-0,3$ & 151,1 & $-0,1$ \\
\hline 16 & 155,4 & 155,0 & $-0,3$ & 155,2 & $-0,1$ \\
\hline 17 & 159,4 & 158,9 & $-0,3$ & 159,2 & $-0,1$ \\
\hline 18 & 163,0 & 162,6 & $-0,2$ & 163,0 & 0 \\
\hline 19 & 165,5 & 166,3 & $+0,5$ & 166,6 & $+0,7$ \\
\hline 20 & 167,0 & 169,7 & $+1,6$ & 170,1 & $+1,9$ \\
\hline
\end{tabular}

$\mathrm{zu}$ machen? Diese Frage glaube ich bejahen zu können, da der neue Vorschlag mathematisch eine Reihe von Vorzügen besitzt:

1. Die neue Funktion gestattet, direkt beliebige Wachstumsvorgänge darzustellen - also auch das Gewichtswachstum - ohne eine Umrechnung auf die Länge vorzunehmen.

2. Es können bei der graphischen Darstellung und der Berechnung direkt die gegebenen Dimensionen eingesetzt werden und es bedarf nicht der Bildung der Differenz von einem Maximalwert. Ebenso ergeben sich bei der Berechnung direkt die Logarithmen der gesuchten Dimensionen. Die neue Formel ist also einfacher in ihrer praktischen Anwendung und erspart eine Reihe von Rechenoperationen.

3. Durch die Möglichkeit mit der neuen Formel beliebige Wachstumsvorgänge darzustellen, kann man ihre Parameter mathematisch in Beziehung zur allometrischen Wachstumsfunktion setzen:

$$
\mathrm{y}=\mathrm{b} \cdot \mathrm{x}^{\alpha}
$$

Es ergibt sich, daß der Exponent $\alpha$ der Quotient der Wachstumsgeschwindigkeiten ist:

Der Faktor $b$ ergibt sich zu:

$$
a=\frac{\log \mathrm{N}_{1}}{\log \mathrm{N}_{2}}
$$

$$
\mathrm{b}=\frac{\mathrm{D}_{\max 1}}{\mathrm{D}_{\max 2^{a}}}
$$


Es läßt sich also aus der neuen Funktion eine sinnvolle Deutung der Parameter der allometrischen Funktion ableiten (KRüGER 1964). Zugleich ist hiermit eine Brücke zu allen Funktionen geschaffen, die der allometrischen Formel folgen.

4. Das in der neuen Formel enthaltene $\zeta$ charakterisiert mathematisch den Wachstumsbeginn. Biologisch wichtiger ist zunächst, daß es einen Ausdruck für den Verlauf der Wachstumskurve darstellt. Ein niedriges $\zeta$ bedeutet ein starkes Anfangswachstum,

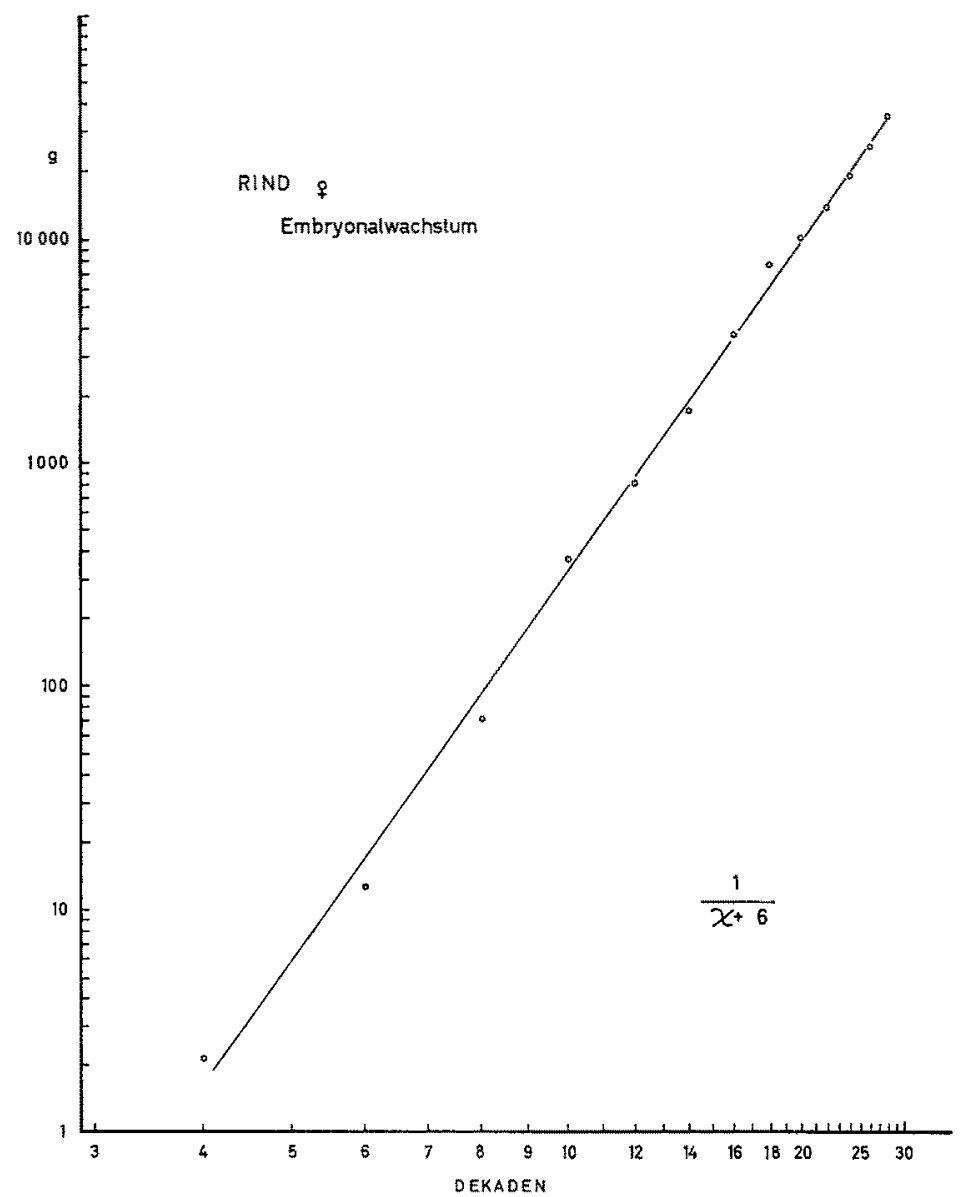

Abb. 6: Graphische Darstellung des embryonalen Wachstums des Rindes (Brody 1945) als Funktion des um 6 Dekaden erhöhten Entwidklungsalters

das sehr schnell abklingt, ein hoher $\zeta$-Wert kennzeichnet einen gleichmäßigeren Wachstumsablauf. Die eingesetzten $\zeta$-Werte in der Dimension von Jahren erscheinen im Augenblick biologisch noch wenig sinnvoll, haben zunächst also nur rein deskriptiven Wert. Sie gelten außerdem nur für das postembryonale Wachstum. Auch das embryonale Wachstum folgt der vorgeschlagenen Funktion, nur liegen in diesem Falle die Werte für $\zeta$ in der Dimension von Wochen oder Monaten. Das eindrucksvollste Beispiel, auf das ich bislang gestoßen bin, stellt das Embryonalwachstum des Rindes dar 
(Brody 1945). Es läßt sich über die Gewichtsspanne von $2 \mathrm{~g}$ bis $35000 \mathrm{~g}$, also über 4 Zehnerpotenzen darstellen (Abb. 6).

5. Die Differentation der neuen Wachstumsgleichung ergibt, daß für die Logarithmen der Wachstumswerte $\log \mathrm{N}$ die Wachstumsgeschwindigkeit darstellt. In die linearen Werte geht außerdem die Maximaldimension ein.

6. Die relativ kurzen Kurvenstücke, die für eine mathematische Analyse der Wachstumskurve zur Verfügung stehen, haben bis heute nicht erlaubt, ihre geometrische Form festzulegen. Aus der logarithmischen Form der neuen Gleichung:

ergibt sich:

$$
\log y=\log D_{\max }-\frac{1}{\chi+\xi} \log N
$$

$$
\log y+\frac{1}{z+\zeta} \log N=\log D_{\max }=\text { Const. }
$$

Wie gut diese Forderung erfüllt ist, geht aus Tabelle 5 für die Wachstums-Werte des Thunfisches hervor. Aus dieser Beziehung ergibt sich, daß die logarithmische Wachstumskurve wenigstens näherungsweise eine gleichseitige Hyperbel darstellt.

Tabelle 5

Konstanz des Produktes aus realisiertem und nicht realisiertem Wachstum für das Längen wachstum von Thunnus thynnus

\begin{tabular}{|cccc|}
\hline Altersklasse & $\begin{array}{r}\log y+\frac{\log N}{\chi+\zeta} \\
=\log L_{\max }\end{array}$ & Alrersklasse & $\begin{array}{r}\log y+\frac{\log N}{\chi}+\zeta \\
=\log L_{\max }\end{array}$ \\
\hline 1 & 2,73410 & 8 & 2,72729 \\
2 & 2,72451 & 9 & 2,72635 \\
3 & 2,71296 & 10 & 2,72305 \\
4 & 2,71296 & 11 & 2,72972 \\
5 & 2,72705 & 12 & 2,72002 \\
6 & 2,72912 & 13 & 2,72335 \\
7 & 2,73082 & & 2,73258 \\
\hline
\end{tabular}

Die hier aufgeführten Punkte, die einen wesentlichen Einblick in die Mathematik des Wachstums gestatten, machen es notwendig, dem neuen Vorschlag Beachtung zu schenken. Zumindest dürtte er geeignet sein, die Diskussion über die WachstumsMathematik wieder in Gang zu bringen, nachdem grundsätzlich erwiesen ist, daß das wichtige Problem des organischen Wachstums einer mathematischen Behandlung zugängig und die ihr von den meisten Biologen entgegengebrachte Skepsis unberechtigt ist.

Als erstes Anliegen meiner mathematischen Formulierungen hatte ich die formelmäßige Beschreibung von Kurvenverläufen aufgefaßt und habe auf einen Weg hingewiesen, der eine mathematische Analyse von unbekannten Kurvenverläufen gestattet. Schon eine mathematisch einwandfreie Beschreibung von Kurvenverläufen ist an sich bedeutungsvoll, da sie größeren Aussagewert hat als unübersichtliche Tabellen und schwer auswertbare Kurven (KrüGER 1963a). Ich bin aber der festen Überzeugung, $\mathrm{da}$ uns eine mathematische Behandlung biologischer Funktionen noch unerwartete 
Aufschlüsse vermittelt. In diesem Zusammenhang möchte ich auf meine mathematische Analyse der Zahlenwerte von Јов (1955) über die Temperaturabhängigkeit der Atmung der Forelle Salvelinus fontinalis hinweisen.

Ich hatte schon früher (1961) die Meßwerte dieser sehr sorgfältigen Untersuchung, die auch der Größenabhängigkeit der Temperaturfunktion Aufmerksamkeit schenkte, einer mathematischen Analyse nach meiner Temperaturfunktion unterzogen. Die Werte von Jов lassen deutlich erkennen, daß die Temperaturfunktion des Stoffwechsels bei diesem Fisch Veränderungen im Verlaufe des Wachstums zeigt. Den Berechnungen wurde damals das Minimum der Quadrate der linearen Werte zugrunde gelegt:

$$
\Sigma\left[y_{i}-\frac{y_{\max }}{1}\right]^{2}=\operatorname{Min} .
$$

Die Auswertung der Zahlen von KroGH führte zu der Erkenntnis, daß man bei dieser Berechnungsweise zu irreführenden Werten kommen kann. Aus diesem Grunde habe ich die Werte von JoB noch einmal durchgerechnet, und zwar mit den logarithmischen Werten, also:

$$
\Sigma\left[\log y-\log \frac{y_{\max }}{\frac{1}{n-z}}\right]^{2}=\text { Min. }
$$

Die auf diese Weise berechneten Parameter (Tab. 6) erfahren vor allem bei den älteren Fischen eine stärkere Veränderung. Dafür ergeben sich aber noch einfachere Verhältnisse für die Größenbeziehung der Parameter. Thre Darstellung im doppelt-

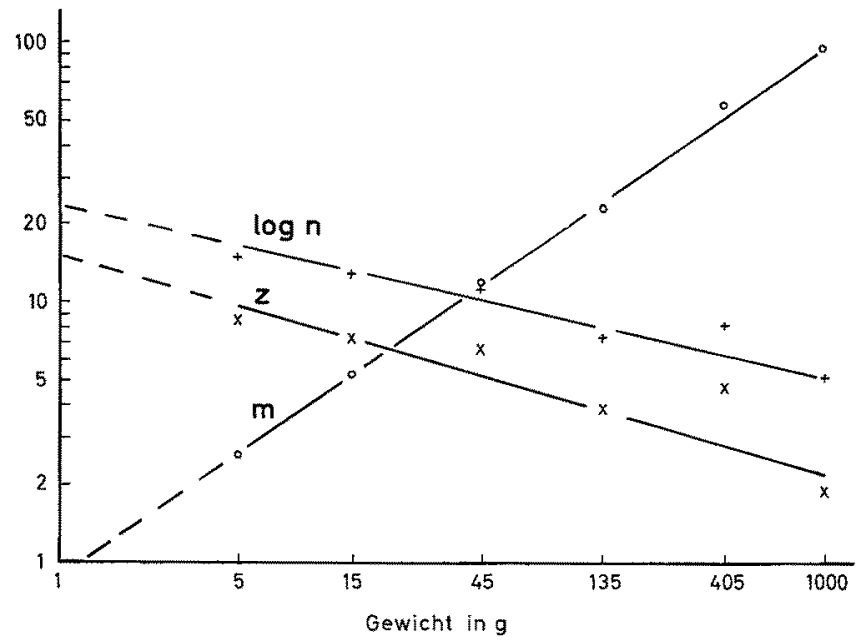

Abb. 7: Doppeltlogarithmische Darstellung der Parameter $\mathrm{m}, z$ und $\log \mathrm{N}$ der Temperaturfunktionen des Fisches Salvelinus fontinalis als Funktion des Gewichtes

logarithmischen Koordinatensystem ergab nach dieser Berechnung für alle drei Parameter nahezu gerade Linien als Funktion des Gewichtes (Abb. 7). Die drei Parameter können also in guter Näherung durch die allometrische Funktion wiedergegeben werden. Nur die Werte für die Fische von $405 \mathrm{~g}$ Gewicht weichen stärker $\mathrm{ab}$ und wurden 
aus diesem Grunde bei der Parameter-Berechnung außer Betracht gelassen. Als Gewichts-Funktion für den Wert $m$ der Gleichung (3) ergab sich:

$$
\mathrm{m}=0,89 \times \mathrm{w}^{0,67}
$$

Da der mathematischen Form nach $m$ ein Ausdruck für Substratkonzentrationen darstellt, steht die Beziehung zur Oberfläche in bester Übereinstimmung mit experimentellen Befunden. Die Deutung der Gewichtsfunktion der beiden anderen Parameter steht noch offen und eribrigt daher eine Diskussion.

Die beiden Konstanten der allometrischen Funktion berechnete ich in der bekannten Weise aus den elektronisch errechneten Parametern (Gleichung 12) der Temperaturfunktion. Die Werte der Funktion für $z\left(z=15,55 \times \mathrm{w}^{0,287}\right)$ eigneten sich wegen der starken Streuung nicht für die Berechnung. Sie wurden in diesem Falle empirisch etwas geändert, um einen besseren Anschluß an die Meßwerte zu erhalten. Die Werte für $m$ und $\log n$ wurden direkt eingesetzt.

Die aus den Versuchswerten von Јов abgeleiteten Parametergleichungen gestatten, die Atmungsgröße von Salvelinus in einem weiten Temperatur- und Gewichtsbereich in sehr guter Näherung rein mathematisch zu berechnen (Tab. 6), einschließlich der $405 \mathrm{~g}$ Fische, die bei der Parameter-Berechnung ausgelassen wurden. Die Abweichungen der berechneten Werte überschreiten in keinem Falle die Fehlergrenze, mit der Atmungsmessungen immer behaftet sind. Da sich die Parameter-Funktionen durch die allometrische Formel darstellen lassen, erscheint es grundsätzlich auch möglich, sie direkt als Funktion des Alters zu berechnen. Leider konnte ich diese Auswertung noch nicht durchführen, da ich noch keine Daten über das zeitliche Wachstum von Salvelinus gefunden habe.

Woran es uns heute noch fehlt, sind Untersuchungen über die Stoffwechselgröße von Organismen als Funktion von Größe, Temperatur und Alter, die den hohen Anforderungen gerecht werden, die eine Bestimmung des Wachstumsparameters verlangt.

Zum Abschluß möchte ich noch kurz auf die Frage eingehen, wodurch sich die formale Ahnlichkeit der Temperatur- und Wachstumsfunktion erklärt. VAN'T HoFF hat die ArrhenIus-Formel als mathematischen Ausdruck eines Gleichgewichtes bezeichnet. Für den Fall der Temperaturfunktion ist das leicht verständlich. In ihr wird der aktive Anteil einer Reaktion durch y repräsentiert, der inaktive Anteil durch den Nenner $n^{1 / t-z}$. Das Produkt beider Anteile ist konstant, nämlich gleich $m$.

Beim Wachstum haben wir es anscheinend ebenfalls mit einem Gleichgewicht $z$ wischen dem realisierten Anteil $y$, der auf die Maximalgröße $D_{m a x}$ tendiert, und dem Nenner $N^{1 / y+\xi}$, der eine das Wachstum hemmende Größe darstellt. Die Verschiebung des Gleichgewichtes erfolgt dann durch eine mit fortschreitendem Alter geringer werdende Wachstumshemmung. Eine ganz entsprechende Deutung enthält übrigens auch die BertalanfFy-Formel.

Meine Ausführungen sollten zeigen, daß es nicht nur möglich ist, biologisch bedeutungsvolle Zahlenbeziehungen durch geeignete mathematische Funktionen zu beschreiben, sondern daß sich darüber hinaus heute schon quantitative Beziehungen zwischen scheinbar ganz heterogenen Faktoren, wie etwa Temperaturabhängigkeit und Alter abzuzeichnen beginnen. In der Verfolgung und weiteren Bearbeitung dieser Probleme liegt ein wichtiges Aufgabengebiet der Stoffwechselphysiologie. 


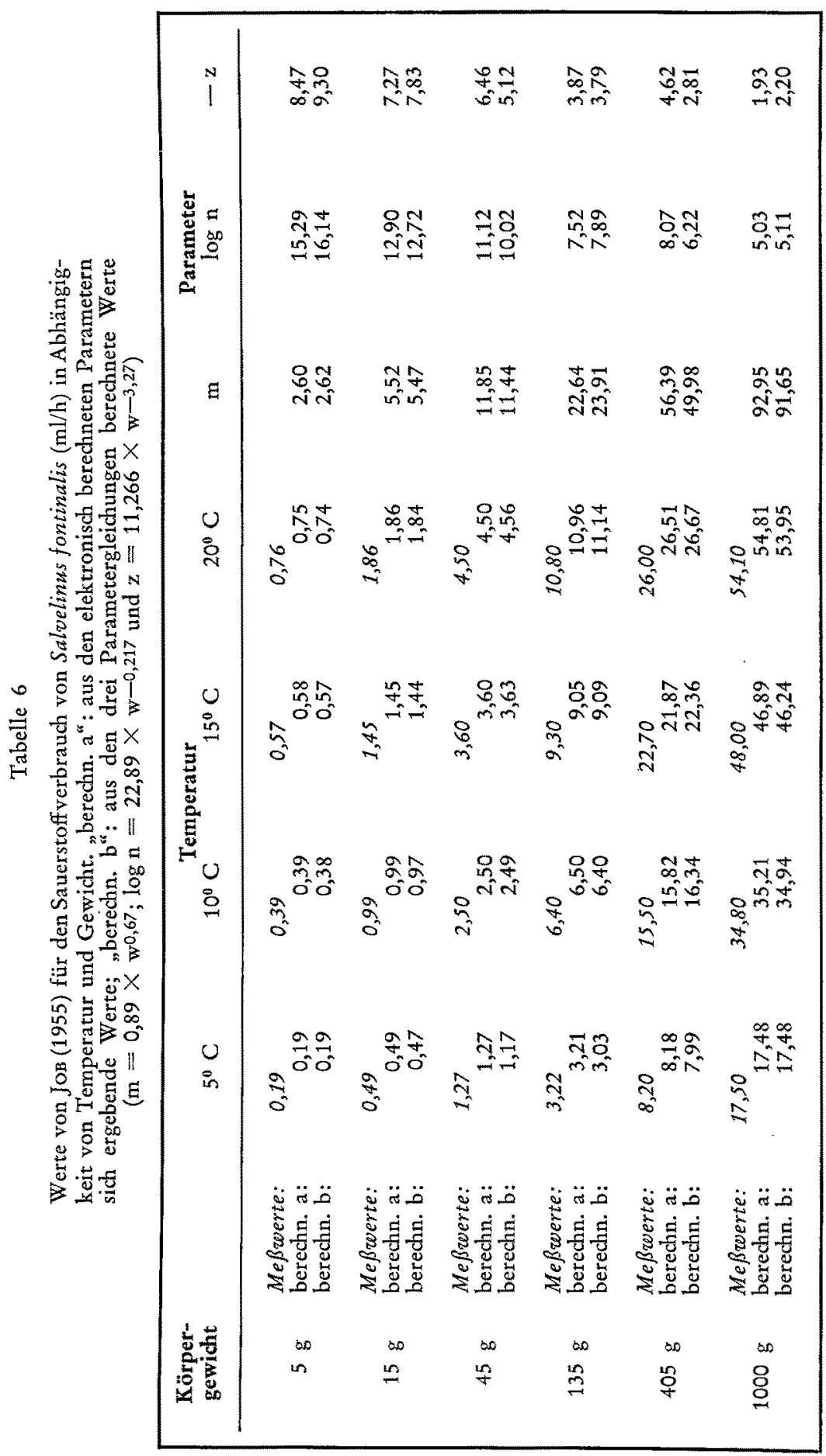




\section{ZUSAMMENFASSUNG}

1. Es wird ein Verfahren für die mathematische Analyse von Kurvenverläufen mit unbekannter Funktion vorgeschlagen. Es beruht darauf, daß man versucht, durch Variation der Koordinatenteilungen die Kurve in eine Gerade überzuführen.

2. Die Anwendung dieses Prinzips auf biologische Temperaturfunktionen führte zu der Formel (3), die auch den Anschluß an die Arrhenrus-Funktion gestattet.

3. Die Bestimmung der Parameter kann graphisch oder auch rein rechnerisch erfolgen.

4. Die Beispiele, die Krogh seiner Standard-Kurve zugrunde legte, lassen sich durch die vorgeschlagene Funktion darstellen.

4. Die Analyse von Wachstumskurven führte zu der mit der Temperaturfunktion identischen Formel (4).

5. Die neue Wachstumsfunktion läßt sich mathematisch leichter handhaben und sich sowohl für Längen- wie auch für Gewichtsdaten anwenden.

6. Die Parameter der Wachstumsfunktion gestatten die mathematische Ableitung der Parameter der allometrischen Wachstumsfunktion: (7) und (8).

7. An den Beispielen des Wachstums von Thunnus thynnus und des menschlichen Längenwachstums zeigte sich eine auffallende Übereinstimmung mit den nach der BERTALANFFY-Formel berechneten Werten.

8. Die Neuberechnung der Parameter für die Meßwerte von Joв (1955) für die Atmung von Salvelinus fontinalis auf der Basis der minimalen Abweichung der Logarithmen der Meßwerte von den Logarithmen der berechneten Werte ergibt bessere Ergebnisse (Tab. 6).

Es ist mir ein tiefempfundenes Bedürfnis auch an dieser Stelle allen denen meinen herzlichsten Dank auszusprechen, die mir bei der Ausarbeitung und Bearbeitung der Berechnungen geholfen haben: Herrn Dr. Nicolovius (Rechenzentrum der Universität Hamburg), Herrn Dr. M. Gillbricht', Fräulein Mührlentramp und Herm P. Pflaum (Biologische Anstalt Helgoland) und Herrn Dipl.-Ing. C. Harder (Hamburg).

\section{ZITIERTE LITERATUR}

Arrhenius, S., 1889. Uber die Reaktionsgeschwindigkeit der Inversion von Rohrzucker durch Säuren. Z. physik. Chem. 4, 226.

Bertalanffy, L. von, 1934. Untersuchungen über die Gesetzlichkeit des Wachstums. 1. Arch. EntreMech. 131, 613-653.

- 1960. Principles and theory of growth. In: Novinsky, W. W., Fundamental aspects of normal and malignant growth, Elsevier, Amsterdam, 156-259.

Berthelot, M., 1862. Essai d'une théorie sur la formation des éthers. Annls Chim. Phys. (Sér. 3) 64, 110-128.

Beverton, R. J. H. \& Holt, S. J., 1957. On the dynamics of exploited fish populations. Fish. Invest. Lond. (2), 19, 1-533.

BRODY, S., 1945. Bioenergetics and growth. Reinhold, New York, 1023 pp.

JoB, S. V., 1955. The oxygen consumption of Salvelinus fontinalis. Univ. Toronto biol. Ser. 61, $1-39$.

JøRGENSEN, N. R., 1916. Undersøgelser over Frequensflader og Korrelation. (Zitiert nach Nielsen, E. T. \& Evans, D. G., Oikos 11, p. 222) Busck, Copenhagen, 214 pp. 
KroGH, A., 1914. The quantitative relation between temperature and standard metabolism in animals. Int. Z. phys. chem. Biol. 1, 491-508.

KRÜGER, F., 1961. Ober den Exponenten der Temperaturfunktion biologischer Vorgänge und deren Größenabhängigkeit. Biol. Zbl. 80, 721-750.

- 1962. Über die mathematische Darstellung des tierischen Wachstums. Naturroissenschaften 49, 454 .

- 1963a. Ein mathematischer Beitrag zum Problem der Temperaturanpassung. Zool. Anz. Suppl. 26, 336-345.

- 1963b. Versuch einer mathematischen Analyse der "Normalkurve" von KroGH. Helgol. Wiss. Meeresunters. 8, 333-356.

- 1964. Mathematische Ableitung der allometrischen Wachstumsfunktion. Zool. Anz. Suppl. 27, 249-253.

Selt.A, M., 1929. Migrazioni e habitat del tonno (Thunnus thynnus) studiati col metodo degli ami, con osservazioni su l'accrescimento etc. (zitiert nach TIEws 1960). Memoria R. Comit. talassogr. ital. 156, 1-24.

TIEws, K., 1960. Bestimmung des Altersaufbaues des roten Thunes (Thunnus thynnus) in der Nordsee etc. Arch. FischWiss. 11, 11-17.

VAN'T HoFf, J. H., 1898. Vorlesungen ïber theoretische und physikalische Chemie. Heft 1-3. Vieweg, Braunschweig, 252, 148, $136 \mathrm{pp}$.

\section{Diskussion im Anschluß an den Vortrag KRüGER}

von Bertalanfry: I need not emphasize how much I agree with Professor Krüger - quite naturally so because we have followed similar lines for well over thirty years. It seems to me that in view of the aims of the present symposium, a certain consideration of principle should be expressed. Physiology nowadays emphasizes "molecular biology", and justly so in view of the great advances made in the fields of nucleoproteins, reaction cycles and many others. However, this emphasis does not make "molar" physiology (to borrow a term used in behavioral science) unnecessary or obsolete, that is, searching for laws at higher levels of organization. This, in fact, is the aim of approaches like those of KRÜGER or myself. With regard to the growth equation proposed by KR ÜGER, I should say that I don't consider my own system as final or exclusive. When confronted with a phenomenon of extreme complexity, such as growth, obviously attack on different lines is in order. KRÜGER's equation has the advantage of easy handling, and so may prove useful, especially if a multiplicity of data should be expressed by few parameters for comparative purposes; although I would emphasize that my system, too, has proved to be useful, particularly in applied fields such as fisheries research (e. g. Bever TON \& Hol. T, 1. c.). My own equations seem to be an aid towards further analysis of processes and limiting factors involved in growth. I am no advocate of "Nothing-but" theories, that is, of the belief that in fields where both complexity of phenomena and our own ignorance are enormous, some monolithic theory should be considered to be the ultimate answer; openmindedness and diligent exploration of possibilities will best promote our field of research.

Krüger: Nach Threr Konzeption schneiden sich bei Auftragung des Aufbau- und Abbaustoffwechsels in einem doppelt logarithmischen Koordinatensystem als Funktion des Gewichtes die Geraden, durch die sie dargestellt werden, in einem Punkt, in dem Anabolismus und Katabolismus gleich groß geworden sind - es ist dieses die Maximalgröße. In meiner neuen Funktion ist die Auftragung in dieser Weise nicht möglich. Die Wachstumsgeschwindigkeit wird in ihr durch $\log N$ gekennzeichnet. Nach meiner Darstellung beginnen Längen- und Gewichtswachstum im Nullpunkt und werden durch zwei divergierende Geraden dargestellt. Aufbau- und Abbauprozesse stehen während des ganzen Wachstums in einem konstanten Verhältnis zueinander. Das Wachstum hört auf, wenn Aufbau und Abbau als Funktion der Zeit gleich Null geworden sind. 
DeHNeL: Have you by badk calculation determined the theoretical rate of growth from time zero?

KRüGer: Die Berechnung des Wachstums auf den Zeitpunkt Null ist nicht möglich, weil die eingesetzten Parameter nur für das postnatale Wachstum gelten. Das embryonale Wachstum folgt zwar der gleichen Funktion, aber mit anderen Parametern. Der $\zeta$-Wert für das embryonale Wachstum liegt in der Größenordnung von 1 bis 2 Monaten.

Honendorf: Durch die Transformationen $\mathrm{N}=$ e $^{\mathrm{k}}$ und $\chi+\xi=\mathrm{t}$ läßst sich die aufgestellte Wachstumsgleichung auf die Form $y(t)=y_{\infty} e^{\frac{k}{t}}$ (mit $D$ max $=y_{\infty}$ ) bringen. In einem t, y-System stellt diese Funktion eine im Nullpunkt beginnende Sigmoidkurve mit einer Asymptote $\mathrm{y}=\mathrm{y}_{\infty}$ dar. Der Wendepunkt dieser Kurve liegt bei $\frac{\mathrm{k}}{2}$ und ist als Umschlagszeitpunkt zwischen dem exponentiell ansteigenden und sich verlangsamenden Wachstum zu deuten. Der y-Wert dieses Punktes beträgt generell für sämtliche Kurven dieser Funktion $\mathrm{y}_{\infty} \cdot \mathrm{e}^{-2}$, also etwa $1 / 7$ des Maximalwertes $\mathrm{y}_{\infty}$. Soweit ich den bisher nach KRÜGERs Formel durchgerechneten Beispielen entnehmen $\mathrm{kann}$, liegt dieser Umkehrpunkt in den meisten Fällen offenbar vor dem "praenatalen Alter" $\zeta$. Von diesem Zeitpunkt an entspricht der Funktionsverlauf einer Wachstumskurve nach voN BERTALANFFY $1(t)=1_{\infty}\left(1-\mathrm{e}^{-k}\left(t-t_{0}\right)\right)$, worauf auch die gleichsinnigen Abweichungen zwischen den nach den beiden Formeln berechneten und den beobachteten Werten in den erwähnten Beispielen hinweisen. Die Ahnlichkeit zwischen der von KRUtGRR auf empirischem Wege gefundenen und der theoretisch abgeleiteten Wachstumsfunktion vON BERTALANFFYs scheint mir eine Bestätigung für die Untersuchungen der theoretischen Biologie zu sein.

VON BERTALANFFY: Of course, a detailed mathematical analysis and comparison of both formulas would be useful. Intuitively, one would expect that they are closely related and in some way isomorphic, and Dr. HoHendorf's treatment indicates an exact way to prove this.

KRÜGER: Ich sehe in der von Herrn HOHENDORF durchgeführten Umwandlung meiner Wachstumsformel in eine e-Funktion vorläufig keinen Fortschritt. Es handelt sich hierbei um eine mathematische Routine-Arbeit, die den klaren Aufbau meiner Funktion verdunkelt. Für die Hand des Biologen erscheint mir die Berechnung mit dekadischen Logarithmen, die ihm jederzeit zur Verfügung stehen, wesentlich einfacher. Der Wert $\chi+\zeta$ für den Wendepunkt liegt bei:

$$
\frac{\ln N}{2}=1,1513 \cdot \log N
$$

Nach den wenigen bislang von mir in dieser Richtung ausgewerteten Zahlenreihen scheint in der 'Tat das maximale Längenwachstum vor der Geburt zu liegen. Ich hatte aber schon 1962 darauf hingewiesen, daß der für das postnatale Wachstum einzusetzende $\zeta$-Wert keine Geltung für das embryonale Wachstum hat. Man kann und darf nicht den $\zeta$-Wert mit der embryonalen Entwicklungszeit identifizieren. Für letzteres gelten andere Parameter. Rein mathematisch kann das maximale Wachstum nur bei $\chi$-Werten liegen, die kleiner sind als $\zeta$. Beim Gewichtswachstum fällt das Maximum nach meinen Erfahrungen in vielen Fällen in das postembryonale Wachstum. Hier ergab sich Obereinstimmung zwischen berechneten und beobachteten Werten. Meine Funktion schließst also auch die Lage des Wendepunktes ein. Vor allem ist an ihr aber wichtig, daß sie relativ leicht einer mathematischen Auswertung zugängig ist. Die neue Funktion enthält auch den aus der allometrischen Funktion abgeleiteten Ansatz der BerTaLANFFYFormel - vermutlich aber in etwas anderer Gestalt. Hier ist vorauszusehen, daß die mathematische Analyse von Wachstumsvorgängen auf Grund der empirisch abgeleiteten Funktion der theoretischen Biologie wichtige neue Gesichtspunkte liefert. 